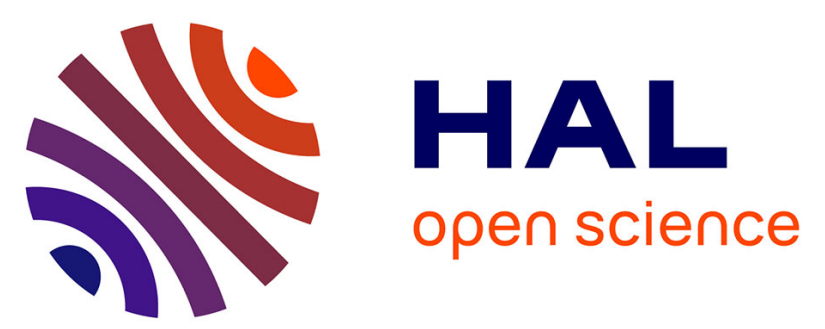

\title{
LA SUPPLICATION COMME RITUEL CHEZ HOMĖRE: LE GESTE ET LA PAROLE
}

Françoise Letoublon

\section{- To cite this version:}

Françoise Letoublon. LA SUPPLICATION COMME RITUEL CHEZ HOMÈRE: LE GESTE ET LA PAROLE. Barbara Cassin; Carlos Lévy. Genèses de l'Acte de parole dans le monde grec, romain et médiéval, études réunies et éditées sous la direction de Barbara Cassin et Carlos Lévy, Brepols, p. 11-28. , Brepols, p. 11-28, 2011, Genèses de l'Acte de parole dans le monde grec, romain et médiéval, études réunies et éditées sous la direction de Barbara Cassin et Carlos Lévy, Brepols, p. 11-28. hal-01276136

\section{HAL Id: hal-01276136 https://hal.science/hal-01276136}

Submitted on 18 Feb 2016

HAL is a multi-disciplinary open access archive for the deposit and dissemination of scientific research documents, whether they are published or not. The documents may come from teaching and research institutions in France or abroad, or from public or private research centers.
L'archive ouverte pluridisciplinaire HAL, est destinée au dépôt et à la diffusion de documents scientifiques de niveau recherche, publiés ou non, émanant des établissements d'enseignement et de recherche français ou étrangers, des laboratoires publics ou privés. 
Je cherche à montrer ici que certains actes religieux tels que prière et supplication obéissent dans l'épopée homérique à des formes très particulières tant dans les paroles prononcées que dans les gestes accomplis. Ces formes particulières impliquent l'existence dans la langue grecque de l'épopée de rituels langagiers spécifiques, que nous mettons en relation avec la théorie des "actes de langage" chez Austin et ses héritiers: il s'agit parfois de performatifs, mais la complexité des relations paradigmatiques attestées dans la langue entraîne un retour sur le détail de la théorie et ses conséquences ${ }^{1}$. En particulier, il faudra réfléchir sur l'importance des gestes et de leur statut dans le développement linguistique du performatif, en synchronie et en diachronie.

La question de la supplication et de la prière en grec a été abordée en français par Danielle Aubriot et Jean Rudhardt dans la même année 1992, ce qui implique évidemment que les auteurs n'ont pas pu tenir compte de la publication de l'autre. Il faut ajouter plusieurs publications en anglais, sur la supplication un article important de Gould publié en 1973 et republié avec un addendum dans un recueil de l'auteur en 2001, puis un livre de Fred Naiden en 2007, ainsi que sur la prière un livre de Simon Pulleyn publié en 2007. Un ouvrage sur la supplication montre son importance dans l'ensemble de l'épopée homérique d'un point de vue plus "littéraire"2, et une spécialiste italienne a publié la même année (1999) un travail anthropologique sur la supplication homérique et un travail sur la "parole efficace" dans le cas de la malédiction, du serment et de la bénédiction dans la Grèce archaïque ${ }^{3}$. Je dirai d'emblée que la thèse de $\mathrm{D}$. Aubriot me paraît faussée par son parti pris paradoxal de départ: elle

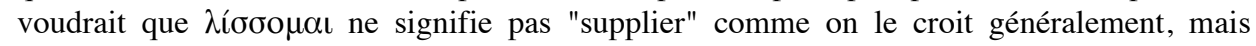
désigne une sorte de prière; elle discute pour cela sur des points mineurs de mon article de $1980^{4}$, mais ignore l'article important de Gould que j'y citais pourtant explicitement, article

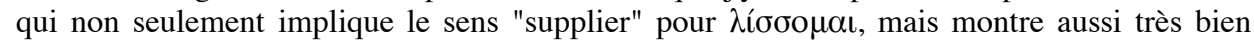
l'importance du rituel de supplication qui me paraît capitale. L'analyse très détaillée que fait Danièle Aubriot des exemples homériques provoque une sorte d'illusion d'objectivité assez troublante à mes yeux quant à la méthode scientifique attendue dans une telle étude.

Nous partirons de l'opposition entre deux types de rituels, de prière et de supplication, qui s'expriment avec des gestes très différents et spécifiques, souvent représentés dans l'iconographie et auxquels nous trouverons de fréquentes allusions dans le texte homérique: la

\footnotetext{
${ }^{1}$ Aux références données antérieurement dans mes travaux sur la question, il faut désormais ajouter les articles "Acte de langage" par B. Cassin, I. Rosier-Catach et S. Laugier, dans le Vocabulaire européen des philosophies, p. 11-21 et "Anglais. La langue anglaise ou le génie de l'ordinaire" par J.-P. Cléro et S. Laugier, III Le paradigme austinien: langage ordinaire et philosophie, p. 95-99.

${ }^{2}$ K. Crotty, The Poetics of supplication. Homer's Iliad and Odyssey, Ithaca, Cornell University Press, coll. Myth and Poetics, 1994.

${ }^{3}$ Manuela Giordano La Supplica. Rituale, istituzione sociale e tema epico in Omero, Napoli, AION 3, 1999; La parola efficace. Maledizioni, giuramenti e benedizioni nella Grecia antica, Pisa-Roma, Istituti editoriali e poligrafici internazionali, 1999.

${ }^{4}$ F. Létoublon, "Le vocabulaire de la supplication en grec: performatif et dérivation délocutive", Lingua 52, 1980, p. 325-336, discuté par D. Aubriot -Sévin, Prière et conceptions religieuses en Grèce ancienne jusqu'à la fin $d u V^{e}$ siècle av. J.-C., Lyon, Maison

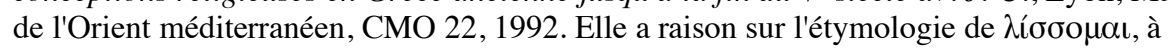
propos de laquelle elle rappelle à juste titre les remarques faites par Benveniste dans son Vocabulaire des institutions indo-européennes. Mais le point central de mon article n'est pas là, mais dans la dérivation du nom du suppliant par rapport à une forme verbale très particulière. M. Giordano a au contraire bien compris ce point central.
} 
prière se fait debout, bras levés vers le ciel, alors que dans la supplication, le suppliant touche les genoux, parfois aussi le menton de la personne qu'il supplie. Comme Pulleyn et Naiden le montrent bien, la prière implique une relation verticale des hommes vers les dieux, in absentia, alors que la supplication implique une relation horizontale in praesentia. La supplication peut aussi passer par l'intermédiaire des dieux, dont on touche l'autel, geste dont les Suppliantes d'Eschyle donnent un très bel exemple. Zeus est le protecteur des suppliants déjà dans l'épopée homérique, on verra plus loin un passage de l'Odyssée dans lequel son épiclèse Hikèsios est précisément mentionnée. Dans le vocabulaire, à l'époque archaïque, il

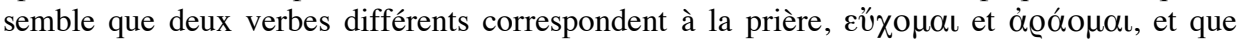

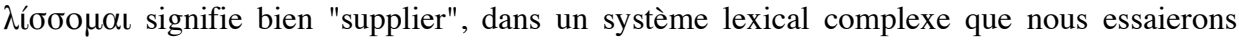
d'analyser en détail.

Il faut aussi parler dès l'introduction de la méthode de l'analyse linguistique, qui repose sur des relevés de tous les emplois d'un terme donné dans un état de langue, le grec archaïque d'Homère en l'occurrence. L'analyse dénuée de préjugé de tels corpus donne parfois des résultats surprenants, même si l'on connaît le grec homérique, par rapport aux données lexicologiques habituelles: les dictionnaires, même les mieux informés comme le LiddellScott, attribuent parfois à Homère des sens qui sont connus dans la langue classique, mais qui s'y sont développés à partir d'interprétations d'Homère, reposant donc sur une forme d'illusion rétrospective de la langue difficile à débusquer: nous l'avons montré pour l'emploi réputé "euphémique" du verbe oĺxoual "être parti": aucun des exemples homériques ne montre en réalité cette valeur euphémique pour "être mort" ${ }^{5}$, mais les deux occurrences dans lesquelles le verbe est employé dans son sens propre d'"être parti" pour parler d'une âme qui a quitté le corps ont fait croire qu'il s'agissait d'un tel emploi. Concernant le verbe "courir", nous avons

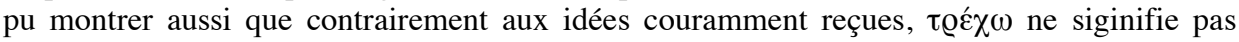
"courir" mais "tourner" chez Homère, le paradigme de "courir" étant constitué par $\theta \varepsilon ́ \omega$ -

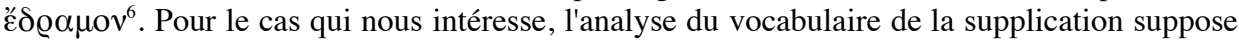
au préalable la connaissance du sens du verbe bâti sur ix-, que sa syntaxe particulière oppose

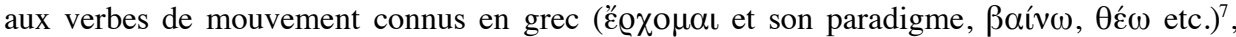

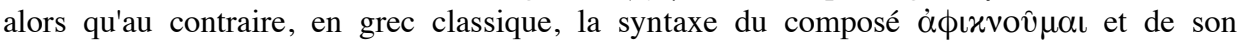
paradigme l'a sémantiquement rapproché des verbes de mouvement au point qu'il semble en être devenu pratiquement un synonyme.

Puisqu'ils impliquent un accord social dans une communauté donnée, les rituels se traduisent par une répétition fréquente de gestes et de paroles, formules magiques ou autres, indépendamment de critères littéraires. Le fait que nos plus anciens textes (en Inde aussi bien que dans la tradition grecque) soient de nature poétique -et plus ou moins religieuse-, avec une grande importance de la forme et particulièrement du vers traditionnellement utilisé dans le genre amènent à un problème: les répétitions formelles rencontrées dans l'Iliade, l'Odyssée, les Hymnes Homériques, la Théogonie, les Travaux et les jours et d'autres œuvres mineures sont-elles le reflet de rituels sociaux contemporains? Ou au contraire, le style poétique a-t-il contribué à créer à partir d'une tradition formulaire des artefacts poétique entièrement artificiels? La réponse doit évidemment être entre les deux, avec une infinité de nuances. Quand on rencontre une association régulière de gestes et de paroles identiques qui ont l'air de formules, on peut conclure qu'un rituel justifie cette coïncidence, surtout dans le cas d'un ton fortement teinté de valeur religieuse (par exemple avec un discours à un dieu ou aux dieux).

\footnotetext{
${ }^{5}$ Voir F. Létoublon, "Ce qui n'a plus de nom dans aucune langue", RPh. 66, 1994, p.317-335.

${ }^{6}$ F. Létoublon, "La roue tourne", en collaboration avec C. de Lamberterie, Rph. 54, 2, 1980 , p. 305-326.

${ }^{7} \mathrm{~F}$. Létoublon, Il allait, pareil à la nuit. Les verbes de mouvement en grec: supplétisme et aspect verbal, Paris, Klincksieck,1985.
} 
Dans certains cas, plusieurs rituels sont associés dans une sorte de Suite à la fois narrative et rituelle. ${ }^{8}$ Nous nous intéresserons particulèrement à ces types d'associations.

La théorie des Actes de langage (Speech Acts ${ }^{9}$ ), depuis Austin ${ }^{10}$, s'est fondée sur une analyse synchronique des langues vivantes - et à vrai dire principalement de l'anglais - avec une visée universelle. L'histoire des langues est rarement invoquée, et il peut sembler incongru et paradoxal d'utiliser des témoignages de langues anciennes et "mortes", sans compréhension orale où que que ce soit, pour montrer qu'on faisait quelque chose en les parlant, pour renvoyer au fameux titre d'Austin. Nous n'essaierons nullement de "prouver" la théorie, que ce soit la version d'Austin ou une autre, en analysant la langue grecque. L'objectif est plutôt de montrer que certains phénomènes dans cette langue peuvent contribuer à soutenir certaines hypothèses venues de la linguistique générale, et parfois d'amener à préciser certains points de vue théoriques. Plusieurs phénomènes apparemment disjoints pourraient, si l'on admet certains postulats théoriques, recevoir une explication générale cohérente. La langue grecque était utilisée, entre autres, pour faire certains actes rituels, et cet usage résonne dans la langue elle-même à travers des procédés particuliers reflétés par le lexique, la morphosyntaxe ou des évolutions sémantiques. On verra aussi que sur plusieurs points, les formulations d'Austin ont entraîné des confusions ou des ambiguïtés sur lesquelles il faut réfléchir à la lumière de nombreux exemples et sans $a$ priori, en tenant compte des conditions d'emploi, du contexte, de la distinction entre récit et discours...

\section{Les formes de la supplication en grec homérique}

La combinaison dans le texte de formules répétées décrivant un geste et/ou rapportant des paroles de supplication adressées par un personnage à un autre apparaît dans le passage suivant: en Iliade I, Achille demande à sa mère la déesse Thétis de supplicr Zeus en sa faveur $^{11}: 1.497-512$, voir en partıculier 500-501 où Thétis fait les actes prescrits:

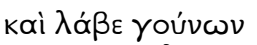

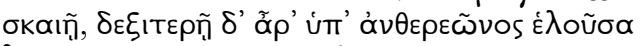

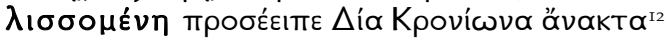

[Thétis] lui saisit les genoux

de sa main gauche, et, le prenand de sa droite au menton,

elle adressa cette prière à Zeus, fils de Cronos ${ }^{13}$.

\footnotetext{
${ }^{8}$ Voir Létoublon, "Comment faire des choses avec des mots grecs. Les actes de langage dans la langue grecque", in Philosophie du langage et grammaire dans l'Antiquité. Cahiers de philosophie ancienne 5, Cahiers du groupe de recherche sur la philosophie et le langage 6-7, Bruxelles-Grenoble,1986, p. 67-90.

${ }^{9}$ La terminologie des actes de langage (Speech-acts) vient de Searle plutôt que d'Austin qui parlait plutôt de faire avec des mots (Dire c'est faire dans l'édition en français, 1970). Voir Lyons sur ce point ainsi que l'encadré dans le Vocabulaire européen des philosophies, p. 20 : la traduction française par langage plutôt que parole ou discours pourrait corriger une erreur dans le choix terminologique. Sur le rôle d'Austin dans le développement de la branche linguistique appelée pragmatique, voir aussi F. Latraverse, La pragmatique, Bruxelles, Mardaga, 1987, p. 32-36.

10 Austin 1962.

${ }^{11}$ Iliade I, 497-512.

${ }^{12}$ Vers 500-502.

${ }^{13}$ La traduction de l'Iliade citée dans cet article est celle de Fr. Mugler (Arles, Actes Sud, 1995) sauf mention explicite.
} 
Comme ses paroles ne persuadent pas le dieu suprême, elle prononce un autre discours $^{14}$, qui entraîne la colère de Zeus (óx $\theta$ ńøas) jusqu'à ce que finalement il accède à sa

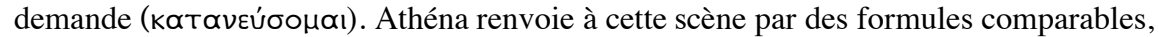

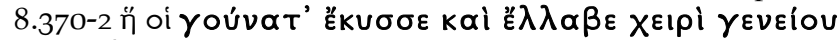

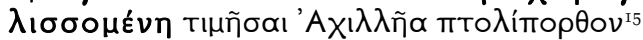

lui baisant les genoux et le prenant au menton,

elle l'a supplié d'honorer le vaillant Achille,

ainsi que Zeus lui-même

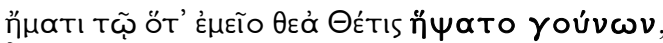

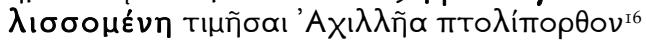

Le jour où Thétis la divine saisit mes genoux,

En m'implorant d'honorer Achiille, ce preneur de villes.

On note que 15.76 est différent de 8.370 alors que 15.77 est identique à 8.371 . Le point essentiel est que le même geste de prendre les genoux d'une main ${ }^{17}$, le menton de l'autre, est exprimé de trois maniètes différentes, alors que l'allusion aux mots prononcés se fait trois fois par la même forme verbale, dont deux par le même vers formulaire ${ }^{18}$. Mais le plus important est le fait que nous n'avons jamais les paroles adressées à Zeus par Thétis: dans le premier cas, le geste et la parole sont rapportés à la troisième personne par le "narrateur objectif" de l'Iliade, dans le deuxième, c'est la déesse Athéna qui rappelle l'attitude de Thétis, bien qu'elle n'ait pas assisté elle-même à la scène; et c'est Zeus qui la rappelle dans la troisième occurrence. Ainsi, on ne rencontre pas ici l'acte de supplication accompli (performed) comme tel, mais rapporté dans le récit. Bien que Danielle Aubriot ${ }^{19}$ n'admette pas

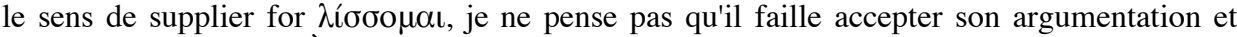
maintiens ce sens ${ }^{20}$. À l'inverse du cas précédent, en Il.18.457-60 aucun geste n'est explicitement rapporté dans le récit du narrateur; mais le long discours tenu par Thétis à Héphaïstos montre bien l'usage du vocabulaire de la supplication, et même une formule orale qui semble accomplir la supplication par des mots, proche de la définition par Austin du performatif:

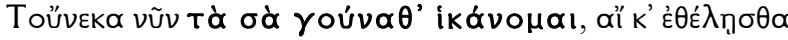

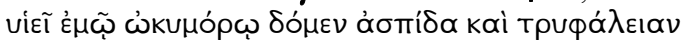

$[\ldots]^{21}$

C'est pourquoi je touche tes genoux. Voudras-tu

Donner à mon fils à la vie brève un bouclier, un casque ...

\footnotetext{
${ }^{14}$ Iliade I, 513-516.

${ }^{15}$ Iliade VIII, 371-372.

${ }^{16}$ Iliade XV, 76-77.
}

${ }^{17}$ Le rôle des genoux dans la conception grecque du corps et de l'âme est expliqué par Onians (ch.IV) comme le siège de la génération, en relation avec la racine *gen connue dans $\gamma \varepsilon ́ v o s$,

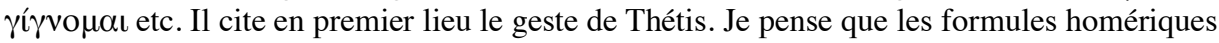
telles que $\lambda u ́ \tau o ~ \gamma o u ́ v \alpha \tau \alpha$ "ses genoux se délièrent", employées au moment de la mort du guerrier ou de la défaillance d'une femme impliquent que les genoux sont pensés comme le siège de la force vitale, voir Richard $\mathrm{B}$. Onians, Les origines de la pensée européenne sur le corps, l'esprit, l'âme, le monde, le temps et le destin, trad. B. Cassin, A. Debru, M. Narcy (éd. orig. en anglais, Cambridge, 1951), Paris, Seuil, 1999.

${ }^{18}$ Voir sur la notion de vers formulaire Milman Parry, The Making of Homeric Verse: The Collected Papers of Milman Parry, ed. and transl. Adam Parry, Oxford, Clarendon Press, 1971.

${ }^{19}$ Voir Aubriot 1992: p. 439-493.

${ }^{20}$ En réalité, elle a à mon avis interprété l'analyse de Benveniste d'une manière particulière, ce qui n'a rien de nécessaire.

${ }^{21}$ Iliade XVIII, 457-458. 
On peut ainsi faire l'hypothèse que les mots attribués à Thétis décrivent le geste accompli, et que le narrateur ne se sent pas contraint de répéter à la fois la description du geste et les paroles. On pourrait conclure, mais il faut rester prudent dans de telles conclusions, que le narrateur traite ici d'un rituel de supplication ${ }^{22}$, et qu'il choisit de garder pour son récit soit la relation des paroles prononcées soit la description des gestes accomplis avec les paroles au discours indirect. L'emploi d'une première personne à l'indicatif présent par Thétis sans mention du geste rituel habituel pourrait constituer le moment fondateur où la parole performative s'émancipe du geste auquel elle est d'abord liée. La proposition au subjonctif introduite par aỉ k' "pour le cas où" implique pour la principale le statut d'un verbe de parole, de même que l'infiniti complément тıñ̄ơı dans l'exemple précédent.

Un autre passage de l'Iliade est capital de ce point de vue, l'épisode de la mort

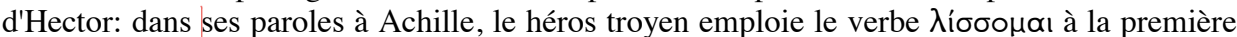

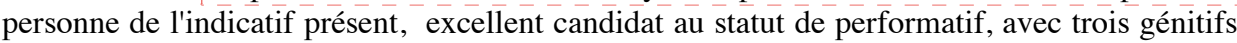
associés qui peuvent impliquer le geste de toucher les genoux, mais avec une valeur rituelle

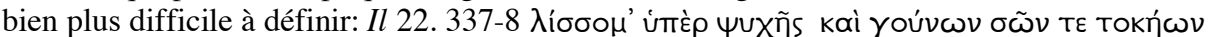

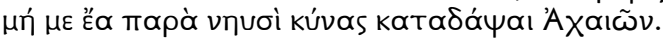

"Je t'en supplie par ta vie, tes genoux et tes parents, ne me laisse pas près des nefs à déchirer par les chiens".

Achille fait écho à ces paroles dans les siennes en reprenant une partie des termes, mais en désignant l'acte d'Hector (apparemment constitué à la fois par un geste non explicite dans les récits et les paroles citées) par un verbe dérivé du nom du genou, 22.345

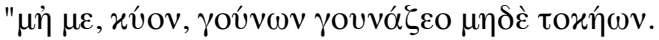

"ne me supplie, chien, ni par mes genoux ni par mes parents".

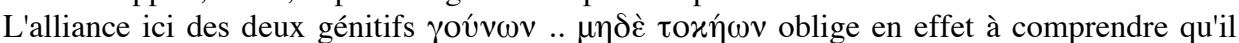
s'agit bien à la fois et par une seule et même forme verbale, de "toucher les genoux" et de "supplier au nom des parents". Une fois encore, le narrateur cite les paroles, mais ne mentionne pas de gestes correspondants: Hector a-t-il touché les genoux d'Achille ou a-t-il seulement dit qu'il les touchait? Le texte ne permet pas de le savoir et finalement, cela importe peu: le rituel a certainement comporté un geste de toucher les genoux, mais la vertu de la parole performative est que la parole prend la place du geste.

Dans le même épisode de la "belle mort" d'Hector, on mentionnera encore l'emploi par Athéna déguisée en frère et allié d'Hector d'un autre dérivé du nom du genou dans le même emploi au sens de "toucher par les genoux", associé à $\lambda i ́ \sigma \sigma o \mu \alpha ı$, parlant des parents d'Hector

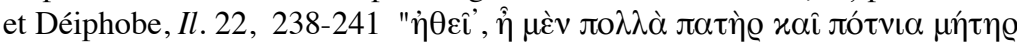

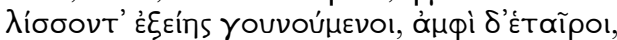

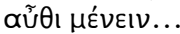

Dans ce cas, l'absence de geste correspondant aux paroles est impliquée par le contexte: les parents d'Hector regardaient le combat du haut des murs de Troie, et suppliaient leur fils de rentrer dans la ville avant qu'il ne soit trop tard.

On rencontre plusieurs occurrences de ce rituel de supplication dans l'Odyssée, car le retour d'Ulysse travesti implique qu'il se présente constamment comme un étranger, un mendiant qui demande l'hospitalité, de sa prière au dieu de la rivière de Schériée ${ }^{23}$ après la tempête à laquelle il a échappé au chant 5; il supplie Nausicaa avec une alternative exprimée

${ }^{22}$ Le rituel a été bien étudié par plusieurs chercheurs, en particulier J. Gould "Hiketeia", JHS 93, 1973, p. 74-103, republié avec un addendum in Gould, Mythn Ritual, Memory, and

Exchange. Studies in Greek Literature and Culture, Oxford, O.U.P., 2001, p. 22-77. Voir F. S. Naiden, Ancient Supplication, Oxford, O.U.P., 2006, avec un intéressant historique de la question.

${ }^{23}$ Odyssée V, 444-450. ire: Ce paragraphe s'est apparemment perdu dans la publication, je le rétablis dans cette version sans toutefois harmoniser la présentation. 


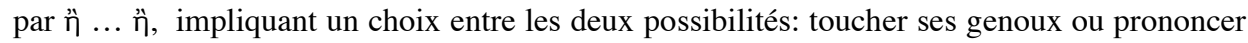
des paroles pour le même acte de supplication, 6.141-185

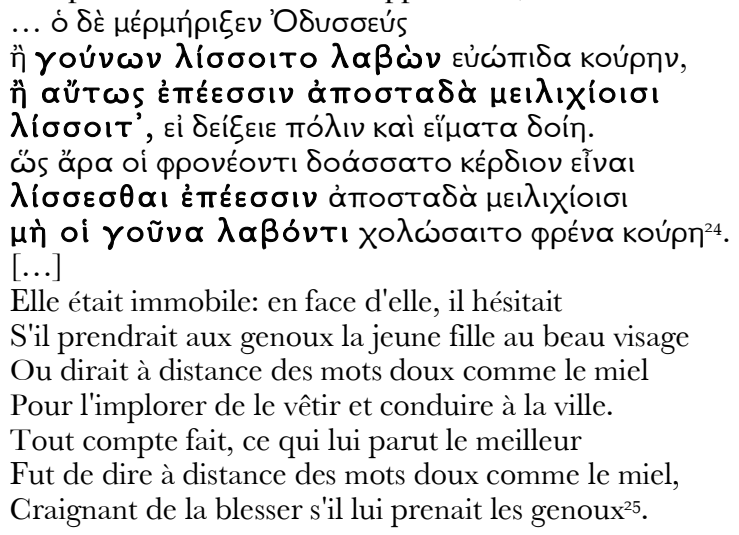

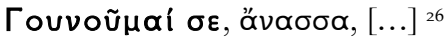

Je prends tes genoux, maîtresse

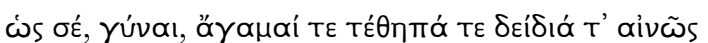

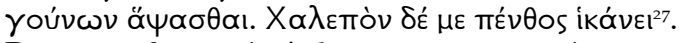

De même, femme, je t'admire avec stupeur, je crains

Infiniment de toucher tes genoux. Ma peine est lourde.

À deux reprises, dans le récit d'abord avec l'expression d'une alternative, puis dans le discours d'Ulysse, on retrouve la même idée: supplier se fait soit en touchant les genoux, soit par des paroles, ou encore par les deux à la fois dans des occasions particulièrement solennelles. Dans le récit, les termes de l'alternative sont surprenants, car la même forme

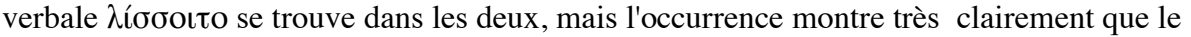

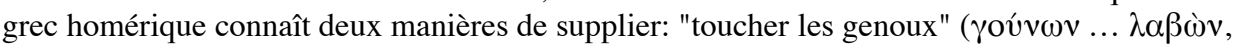

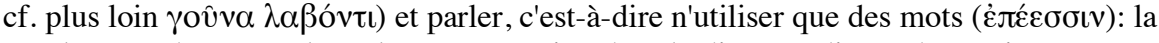
parole peut donc remplacer le geste; ensuite, dans le discours direct, de manière très étonnante, l'usage d'un verbe dérivé du nom du genou youvoũuaı est utilisé pour signifier "supplier", sans développement supplémentaire ${ }^{28}$ et alors même qu'Ulysse dit explicitement qu'il a peur de toucher les genoux de Nausicaa aux vers 168-169.

Le même Ulysse fait plus loin presque la même chose avec la mère de Nausicaa, Arété $^{29}$, mais il lui touche les genoux dans le récit et dit qu'il les touche dans ses paroles:

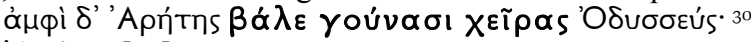

'Apท́тп, [...]

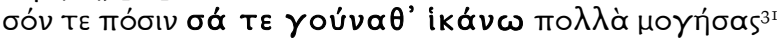

${ }^{24}$ Ibid. VI, 141-147.

${ }^{25}$ La traduction de l'Odyssée est celle de Philippe Jaccottet (Homère, L'Odyssée, Paris, La Découverte, 1982).

${ }^{26}$ Odyssée VI, 149.

${ }^{27}$ Odyssée VI, 168-169.

${ }^{28}$ Sur gounoumai ici, voir Flávio Ribeiro de Oliveira, "Gesto e abstração: usos do verbo gounoûmai em Homero", Trans/Form/Ação, São Paulo, 29(1): 63-68, 2006, en français avec modifications dans Gaia 14, 2011.

${ }^{29}$ Odyssée VII, 142-152.

${ }^{30}$ Odyssée VII, 142.

${ }^{31}$ Odyssée VII, 146-147. 
$[\ldots]$

Ulysse mit ses bras autour des genoux de la reine [...]

Arétè $[\ldots]$

je viens à ton époux, à tes genoux, homme meurtri

On note d'ailleurs dans les paroles d'Alcinoos plus $\operatorname{loin}^{32}$ l'invocation à Zeus comme protecteur des suppliants,

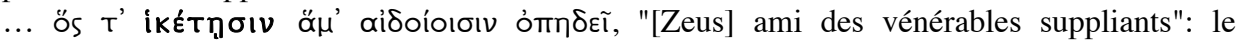
substantif dérivé du verbe fondé sur ik- semble signifier "suppliant" sans problème ${ }^{33}$.

Ces passages montrent bien la complémentarité du système lexical dans une sorte d'association paradigmatique: verbe $\lambda i ́ \sigma \sigma o \mu \alpha ı$ (au moins une fois dans l'emploi apparemment

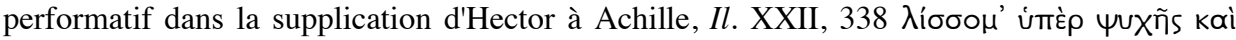

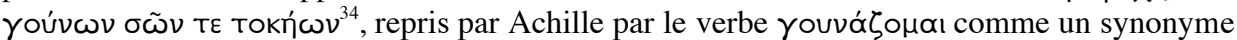

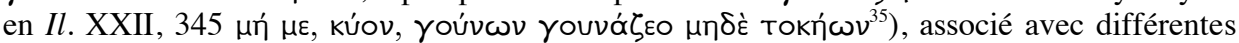
formes du présent de l'indicatif ik-, qui semble insister sur l'importance du corps, et en

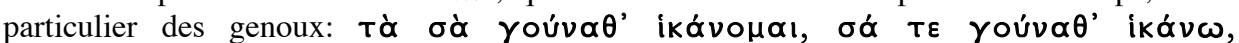

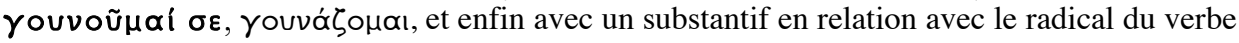
performatif ik-, ikétns.

On peut aussi remarquer que la mention des genoux de l'interlocuteur touchés par le suppliant se rencontre avec d'autres formes verbales (en dehors du présent de l'indicatif), telles

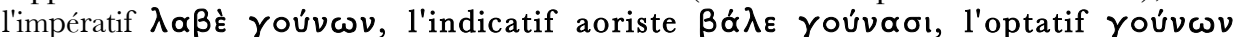
$\lambda i ́ \sigma \sigma o ı t o ~ o u ~ \gamma o u ́ v \omega v ~ \gamma o u v a ́ \zeta \varepsilon o$ dans le langage d'Achille.

Il peut paraître surprenant qu'aucun substantif n'ait été formé en grec pour désigner le suppliant soit à partir de $\lambda \hat{i} \sigma \sigma-$ ou de $\gamma o u ́ v$-.Le seul substantif attesté dans le contexte est

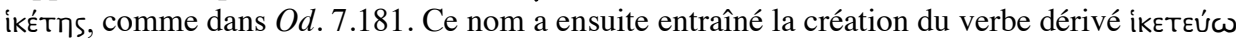
et de toute la famille correspondante, connue avec le sens de supplier en grec classique.

Nous avons proposé ${ }^{36} l$ l'hypothèse d'une dérivation de cette forme de substantif à partir

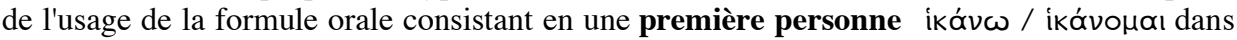
un emploi performatif au sens fort, et de l'accusatif direct du nom des genoux, yoúvata,

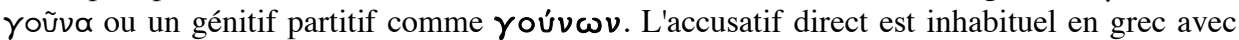
un verbe de mouvement; c'est pourquoi implique ici une autre valeur, proche de celle de "toucher", supposant un contact avec l'objet ${ }^{37}$. Ce point est très important, il n'a pas été compris ni pris en compte par D. Aubriot: c'est à cause de sa construction syntaxique que dans la "synchronie homérique", ik- ne peut pas signifier "arriver, atteindre" comme on le croit généralement, en fonction d'une illusion rétrospective due à la meilleure connaissance que l'on a de la langue grecque de l'époque classique. Effectivement, comme cela a été montré dans ma thèse, ix- a évolué après Homère vers un sens de verbe de mouvement, mais l'ensemble de ses emplois homériques implique qu'il a chez Homère le sens d'un verbe de contact, non de mouvement. Il faut donc le comprendre comme "toucher" plutôt qu'"arriver à".

\footnotetext{
${ }^{32} \mathrm{Au}$ chant VII, vers 181.

${ }^{33}$ Et c'est l'ensemble cohérent de ce système qui me semble invalider la conclusion de D. Aubriot, d'abord séduisante dans sa fidélité à Benveniste et dans l'abondance de ses analyses. 34 "Je te supplie par ton âme, par tes genoux et par tes parents".

35 "Ne me prends pas par mes genoux, chien, au nom de mes parents" (je traduis le plus littéralement possible.

36 "Le vocabulaire de la supplication en grec ...", art. cit.

${ }^{37}$ Benveniste a déjà noté la relation entre í- et íḱtns, mais il dépendait de l'interprétation courante de la forme verbale comme "arriver". Mon hypothèse repose sur la démonstration selon laquelle la syntaxe de l'accusatif direct implique un autre sens, "toucher" (Létoublon 1985).
} 
La dérivation du nom íćtns implique que le sens de supplication ne provient pas du sens du verbe lui-même, mais de l'utilisation rituelle de la formule "je touche tes genoux", qui décrit le geste de supplication, comme on peut le voir sur de nombreux exemples de peintures de vases ou d'autres objets d'art. Dans l'article mentionné, j'insistais sur l'usage performatif de cette formule, et j'ai proposé une dérivation délocutive (suivant une hypothèse de Benveniste en 1958 pour expliquer des termes comme lat. parentare, salutare: dire Parentes, salus etc.. Mais il ne parlait que de verbes délocutifs, la dérivation d'un substantif à partir d'un verbe performatif n'entrant pas dans son objet d'étude).

Dans les exemples homériques relevés, on remarque que les suppliants utilisent deux

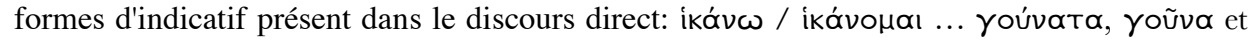

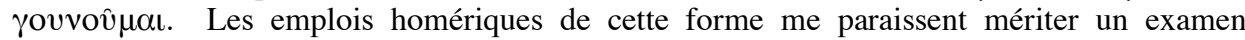
détaillé à l'aide du TLG. Lycaon s'adresse à Achille ${ }^{38}$ :

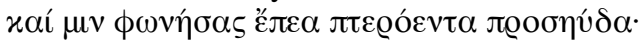

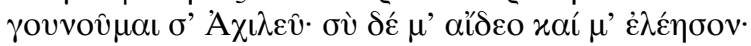

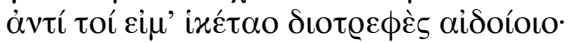

Lors, s'adressant à lui, il lui dit ces paroles ailées:

"Je suis à tes genoux, Achille; écoute et prends pitié!

Respecte en moi, ô nourrisson de Zeus, l'homme qui te supplie."

Dans l'Odyssée, VI,149, il s'agit d'Ulysse face à Nausicaa.

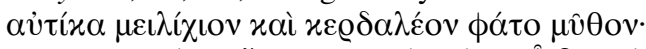

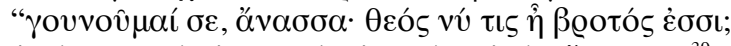

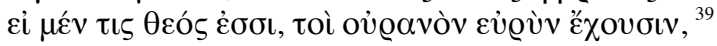
sans attendre, il lui tint cet astucieux discours:

"j'embrasse tes genoux; es-tu femme ou déesse?

Si tu es l'un des dieux qui possèdent la terre immense ..."

Puis de Léiodès face à Ulysse:

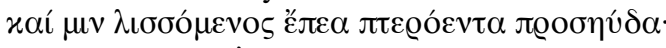

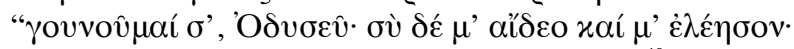

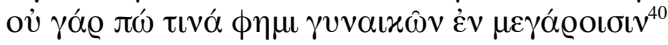

[Léiodès] en le suppliant, lui dit ces paroles ailées:

"j'embrasse tes genoux: entends-moi, aie pitié de moi!

Je l'affirme: jamais je n'ai insulté, outragé les femmes du palais."

Et un peu plus loin, de Phémios:

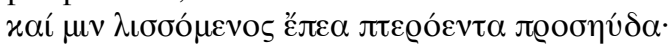

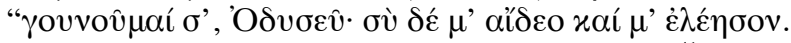

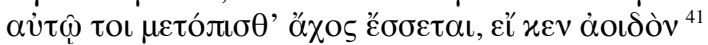

[Phémios] en le suppliant, lui dit ces paroles ailées:

"j'embrasse tes genoux: entends-moi, aie pitié de moi!

Toi-même en auras du regret plus tard, si tu condamnes

Le chanteur..."

Ces vers reprennent de manière formulaire l'emploi d'Iliade XXI, 74.

Dans tous ces exemples, le verbe est en tête de vers, suivi d'un vocatif, nom propre ou appellatif.

On connaît d'ailleurs quelques "citations" de ces emplois: le poète lyrique Anacréon en

présente deux exemples dans ses fragments ${ }^{42}$, Apollonios de Rhodes place la sienne après une

38 Iliade XXI, 73-75.

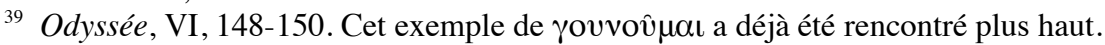

${ }^{40}$ Odyssée XXII, 311-313.

${ }^{41}$ Odyssée XXII, 343-345.

${ }^{42}$ Anacréon, (Page: Poetae melici Graeci) Fr. 3, 3

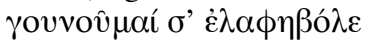


comparaison explicite à Arété et Alcinoos, Argonautica IV, 1014

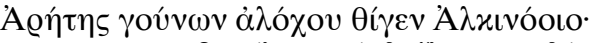

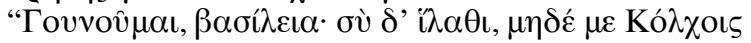

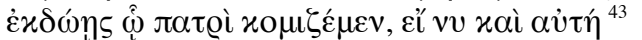

Que de fois elle prenait dans ses mains les genoux d'Arété, l'épouse d'Alkinoos!

"Je suis à tes genoux, Souveraine! Sois miséricordieuse; ne me livre pas aux

Colques qui me ramèneraient à mon père." ${ }^{44}$

Dans d'autres cas, un caractère parodique est possible, très net en tout cas chez Lucien ${ }^{45}$ :

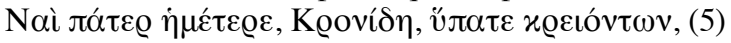

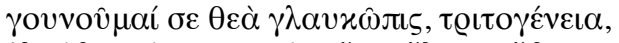

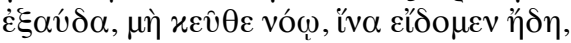

Oui, notre père, fils de Cronos, le plus grand des puissants,

Je te prends les genoux, déesse aux yeux pers, Tritogénie,

Parle, ne me cache rien en ton cœur, pour que nous sachions. ${ }^{46}$

Le verbe est toujours placé en début de vers avec le pronom complément $\sigma$ ' ou $\sigma \varepsilon$, puis des appellatifs de la personne suppliée. Le statut de performatif explicite de cette forme est confirmé par le fait que les autres formes ne sont pratiquement pas attestée: des recherches

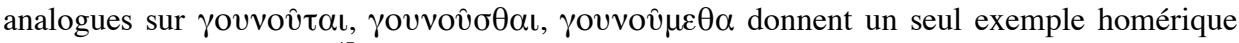
de l'infinitif youvov̂ $\sigma \theta \alpha \iota^{47}$ dans le récit de la Nekyia, et plus précisément dans les instructions de Tirésias, où l'infinitif transpose visiblement au discours indirect un impératif du discours direct, pas une première personne de l'indicatif mais une forme du dialogue impliquant fortement la "personne" au sens dans lequel Benveniste l'oppose à la "non-personne". Après Homère, l'infinitif se trouve dans un exemple du grammairien Apion donnant des glossai homériques, et dans le commentaire à l'Odyssée du savant byzantin Eustathe, ce qui nous confirme bien que le mot n'est pas usité en grec après Homère, sauf citation expresse. La

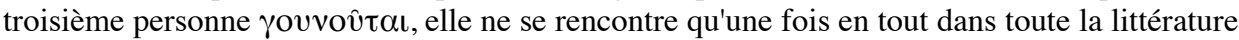
grecque selon le $\mathrm{TLG}^{48}$, chez le même Eustathe, où elle sert de substitut à $\lambda{\text { í} \sigma \sigma \varepsilon \tau \alpha \mathrm{i}^{49}}$.

Nous concluons donc que youvô̂ $\mu \alpha$ joue nettement le rôle de performatif explicite servant à supplier, comme si l'on avait formé en français_un dérivé de genou tel que *genouiller dans ce sens (ce qui n'a rien d'invraisemblable si l'on pense à des expressions telles que "je m'agenouille à tes pieds"). Et il est pratiquement plus fréquent dans cet emploi performatif

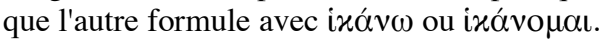

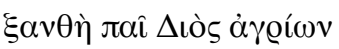

"je te prends les genoux, blonde [déesse] qui lance ses flèches au loin, fille de Zeus..."

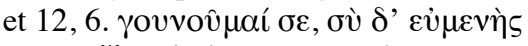

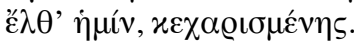

"je te prends les genoux, sois-moi favorable, viens à nous ..."

${ }^{43}$ Arg. IV, 1013-1015. Voir aussi le grammairien de l'époque tardive Apollonius Dyscole, De constructione 2.2 , p. 415, 1.5 où le lemme montre bien que pour lui, il s'agit d'une

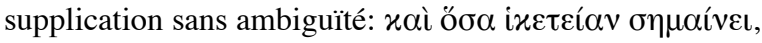

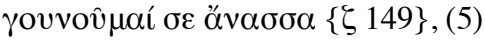

"et tout ce qui signifie la supplication, "je te prends les genoux, maîtresse".

${ }^{44}$ Trad. É. Delage et F. Vian, Belles Lettres, 1981.

${ }^{45}$ Lucien, Juppiter tragoedus 1, 6.

${ }^{46}$ Ma traduction.

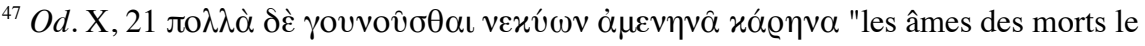
suppliaient abondamment."

${ }^{48}$ Thesaurus linguae graecae.

${ }^{49}$ Comm. Ad Homeri Odysseam I, 398, 1 . 


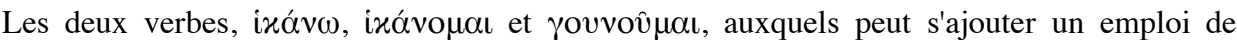
$\lambda i ́ \sigma \sigma \circ \mu \alpha \mathrm{l}$, semblent des exemples parfaits du performatif austinien le plus pur: Austin et Benveniste doivent s'appuyer sur le sentiment de la langue qu'ont les locuteurs pour montrer la différence entre "je jure, je promets" et les autres formes du paradigme, alors que le grec nous montre l'exemple presque idéal d'une langue où l'acte de langage utilise à la première personne du présent de l'indicatif une forme lexicale particulière, mais désigne cet acte dans le récit par un autre lexème.

\section{Le performatif, la théorie et l'histoire de la langue}

Avant d'avancer dans l'analyse des exemples grecs susceptibles d'avoir un retentissement sur la théorie du performatif, je voudrais insister sur le rôle de Benveniste dans la découverte du performatif qu'il a en effet inventé, sans le mot mais bien avec l'idée, avant Austin $^{50}$ : l'article "La philosophie analytique et le langage" publié dans Les études philosophiques en 1963 a été publié après un article d'Austin proposant performatif/constatif que Benveniste connaît et discute, mais on peut pourtant lire une page dans "De la subjectivité dans le langage" qui montre que Benveniste avait découvert la notion dès 1958: il cherche ce qui distingue la "subjectivité", et découvre "les effets de sens que produit le changement de personne dans certains verbes de parole. Ce sont des verbes qui dénotent par leur sens un acte individuel de portée sociale: jurer, promettre, garantir, certifier, avec des variantes locutionnelles telles que s'engager à... se faire fort de ..." On retient la notion d'acte individuel de portée sociale. Le paragraphe suivant comporte la notion suivant laquelle une énonciation est un accomplissement:

'Or je jure est une forme de valeur singulière, en ce qu'elle place sur celui qui s'énonce je la réalité du serment. Cette énonciation est un accomplissement: "jurer" consiste précisément en l'énonciation je jure par quoi Ego est lié. L'énonciation je jure est l'acte même qui m'engage, non la description de l'acte que j'accomplis. En disant je promets, je garantis, je promets et je garantis effectivement. Les conséquences (sociales, juridiques, etc.) de mon jurement, de ma promesse, se déroulent à partir de l'instance de discours contenant je jure, je promets. L'énonciation s'identifie avec l'acte même. Mais cette condition n'est pas donnée dans le sens du verbe; c'est la "subjectivité" du discours qui la rend possible. On verra la différence en remplaçant je jure par il jure. Alors que je jure est un engagement, il jure n'est qu'une descrip^tion, au même plan que il court, il fume. On voit ici, dans des conditions propres à ces expressions, que le même verbe, suivant qu'il est assumé par un 'sujet" ou qu'il est mis hors de la "personne", prend une valeur différente. C'est une conséquence de ce que l'instance de discours qui contient le verbe pose l'acte en même temps qu'elle fonde le sujet. Ainsi l'acte est accompli par l'instance d'énonciation de son "nom" (qui est "jurer"), en même temps que le sujet est posé par l'instance d'énonciation de son indicateur (qui est "je")." 51

\footnotetext{
${ }^{50}$ Avant Austin, on peut mentionner quelques prédécesseurs, le Suédois Hägerstrom et l'Américain Furberg, voir Létoublon "Promisi per iocum" in Studies in Latin Linguistics. R. Coleman ed., Amsterdam,, Benjamins, 1991, p. 163-185. La page de Benveniste de1958 est en général ignorée dans la bibliographie (voir "De la subjectivité dans le langage", Journal de psychologie 1958, repr. in Problèmes de linguistique générale, Paris, Gallimard, 1966, p. 258266).

51 "De la subjectivité dans le langage", Journal de psychologie 1958, repr. in Problèmes de linguistique générale, Paris, Gallimard, 1966, p. 265-266.
} 
Il me semble que l'on peut extrapoler ce que dit Benveniste de jurer et promettre au vocabulaire de la supplication en grec archaïque, avec la seule différence que Homère n'utilise pas les mêmes léxèmes pour l'énonciation réflexive, qui constitue l'accomplissement de l'acte

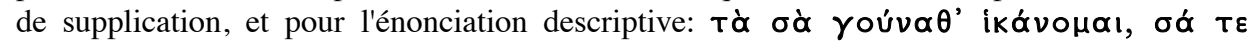

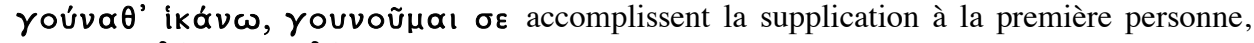

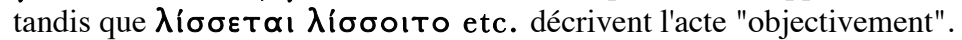

La similitude avec la définition d'Austin est frappante, voir par exemple dans Quand dire c'est faire : "Pour ces exemples, il semble claire qu'énoncer la phrase (dans les circonstances appropriées, évidemment), ce n'est ni décrire ce qu'il faut bien reconnaître que je suis en train de faire ** en parlant ainsi ni affirmer que je le fais: c'est le faire." Et Austin ajoute une remarque importante sur la relation au vrai et au faux; "Aucune des énonciations citées n'est vraie ou fausse: j'affirme la chose comme allant de soi et ne la discute pas. ${ }^{52}$ C'est à ce moment de sa première conférence qu'il crée le terme performatif, ou plutôt phrase performative, insistant sur l'importance de l'énonciation et sur l'aspect créatif et innovant de cette terminologie: c'est pourquoi je pense qu'il ne faut pas diluer le performatif dans un emploi qui engloberait par exemple tout usage poétique et créateur du langage: la poésie crée bien quelque chose, et comporte évidemment des performatifs ${ }^{53}$, sans que pour autant l'ensemble d'un poème puisse être mis sous le signe du performatif comme tendent à le dire d'assez nombreux spécialistes de littérature.

Austin n'a certes pas parlé de la relation entre performatif et rituel ni du rôle des gestes en relation avec les paroles prononcées, mais il insiste beaucoup sur le rôle des "circonstances" :

Disons, d'une manière générale, qu'il est toujours nécessaire que les circonstances dans lesquels les mots sont prononcés soient d'une certaine façon (ou de plusieurs façons) appropriées, et qu'il est d'habitude nécessaire que celui-là même qui parle , ou d'autres personnes, exécutent aussi certaines autres actions -actions "physiques" ou "mentale", ou même actes consistant à prononcer ultérieurement d'autres paroles ${ }^{54}$.

Puisqu'il prend l'exemple assez particulier du baptême d'un bateau, on connaît bien les actions physiques supplémentaires dont il s'agit: couper un ruban, casser une bouteille de champagne sur la coque du bateau etc. Il s'agit bien d'actes rituels à caractère symbolique, qui distinguent par exemple le baptême d'une personne de celui d'un navire. Pour la supplication en grec archaïque, nous avons vu l'importance des gestes rituels accomplis, toucher les genoux ou le menton de la personne que l'on supplie.

Dans sa première conférence, nous rappellerons aussi que Austin a analysé de manière assez détaillée un passage de l'Hippolyte d'Euripide, dont nous avons repéré plusieurs citations ou allusions dans la littérature ancienne ultérieure (Aristophane, Plaute) prouvant la célébrité de ce passage dès l'Antiquité et les interprétations ironiques qu'il a pu susciter, dans lequel il est dit qu'une promesse a été fait par la langue, non par l'esprit:

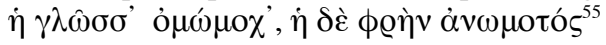

qu'Austin glose ainsi :

"c'est-à-dire "ma langue prêta serment, mais non pas mon cœur (ou mon esprit ou quelque autre artiste dans les coulisses). C'est ainsi que "je promets de... m'oblige: enregistre mon acceptation spirituelle de chaînes non moins spirituelles." ${ }^{56}$

Ce passage d'Austin m'a amenée jadis à étudier de près le cas de la promesse dans l'Antiquité, en particulier en latin, réfléchissant sur l'étymologie de promittere "mettre en

\footnotetext{
${ }^{52}$ P. 41 dans l'édition de 1970, mais voir aussi celle de 1991: John Austin, Quand dire, c'est faire, introduction, traduction et commentaire par Gilles Lane, postface par François Récanari, Paris, Points Seuil, 1991.

${ }^{53}$ Je pense par exemple au $\phi \alpha \mu u$ de Médée au début de la IV ${ }^{\mathrm{e}}$ Pythique de Pindare.

${ }^{54}$ Austin, Quand dire, c'est faire, op. cit. p. 43.

${ }^{55}$ Euripide, Hippolyte, v. 612: "[M]a langue a juré, mais pas l'esprit" (je traduis).

${ }^{56}$ Austin, Quand dire, c'est faire, op. cit. p. 44.
} 
avant", et sur un contresens notable chez certains spécialistes modernes ${ }^{57}$. Un autre cas semble éclairant, celui du grec $\dot{\varepsilon} \gamma \gamma \cup \hat{\omega}$ utilisé en grec classique pour donner sa fille en mariage: chez Hérodote, Clisthène accorde sa fille à Mégaclès fils d'Alcméon avec une première personne du singulier du présent actif que nous interprétons comme un performatif typique: "... $\tau \hat{i} \delta \delta \varepsilon$

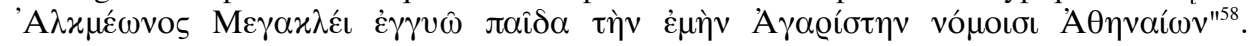
L'acceptation de Mégaclès, au discours indirect, ne permet malheureusement pas de dire avec certitude si la forme médio-passsive correspondante ( $\dot{\varepsilon} \gamma \gamma \cup \omega \mu \alpha$, voir des emplois analogues

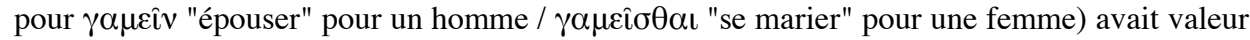
d'engagement pour le "fiancé", mais le laisse au moins supposer: $\Phi \alpha \mu \varepsilon ́ v o v$ dè $̇ \gamma \gamma v \hat{\alpha} \sigma \theta \alpha \iota$

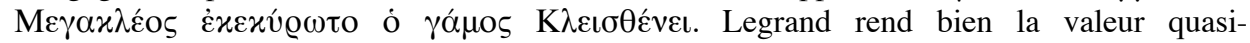
contractuelle des formes verbales: "... Et j'engage mon enfant, Agaristé, au fils d'Alcméon, Mégaklès, conformément aux lois des Athéniens." Mégaclès déclara accepter l'engagement; et ainsi se trouva ratifiée l'union voulue par Clisthène."

Un détour par l'histoire de la langue me semble utile maintenant pour montrer comment les performatifs se développent dans la diachronie, et pour montrer surtout comment ils impliquent des gestes physiques au départ, et tendent à les effacer par une sorte d'incorporation du sens originel dans l'emploi performatif.

En effet, $\dot{\varepsilon} \gamma \gamma \cup \hat{\omega}$ est un composé de la préposition $\dot{\varepsilon} v$ avec un très vieux nom de la main,

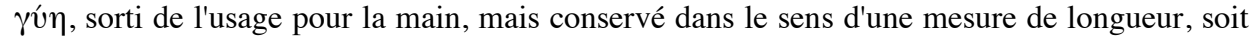
quelque chose comme une "brasse" ${ }^{159}$ : il s'agit donc de s'engager en tenant en main l'objet ou la personne dont il est question. A l'époque d'Hérodote, l'accord entre les deux personnages se fait très probablement sans la présence d'Agaristé, la fille que Clisthène "engage" à Mégaclès. Le français engager est d'une manière très claire formé à partir de en gage, locution encore en usage dans un sens très voisin (donner en gage, tenir en gage etc.).

Le latin promittere implique en fait dans un premier temps, non pas la main droite mais un objet direct "mis en avant" (un ex voto, une somme d'argent, un animal prévu pour un sacrifice ou tout objet que l'on puisse justement engager). A l'époque de Plaute déjà, l'objet de la promesse n'a nullement besoin d'être physiquement présent, la promesse engage l'avenir et implique donc une prise en compte d'un délai entre le temps de la promesse et celui de sa réalisation. Mais l'absence d'emploi performatifs nettement caractérisés empêche d'aller plus loin dans l'analyse.

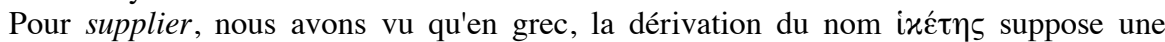

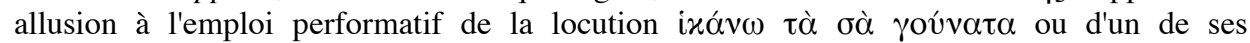
suppléants, donc au geste de toucher les genoux, qui faisait partie du rituel complet de la supplication à l'époque archaïque. Mais avec le temps, le suppliant n'a plus besoin du geste pour que son statut de suppliant soit admis. Tout se passe comme si la valeur performative de

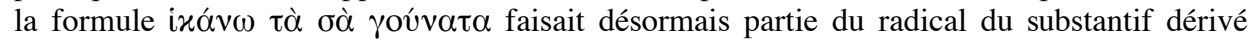
(alors évidemment que le verbe ne connaît plus cet emploi de l'accusatif direct à l'époque classique).

En latin, supplicare semble renvoyer à un geste de prosternation, de "pliure" aux pieds d'une personne (cf. supplex composé comme sim-plex, com-plex etc.).

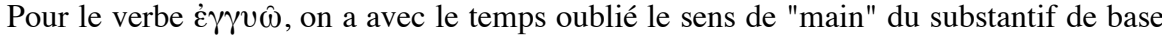

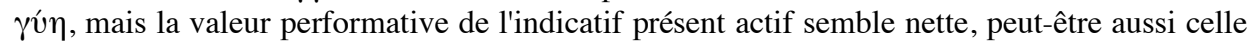
du moyen-passif. Et on n'a évidemment nullement besoin d'avoir dans la main quoi que ce soit en garantie de l'engagement, purement verbal donc.

\footnotetext{
${ }^{57}$ Récanati en particulier pense qu'il s'agit de mettre en avant sa main droite, ce dont une analyse rigoureuse du corpus du latin de l'époque républicaine démontre à l'évidence le caractère erroné.

${ }^{58}$ Hérodote, Histoires VI, 130: "À Mégaclès, fils d'Alcméon, j'engage ma fille Agaristé suivant les lois des Athéniens" (je traduis).

${ }^{59}$ Voir P. Chantraine, DELG s.v.
} 
Dans tous ces exemples, l'emploi performatif semble susceptible de se développer à partir d'une description du geste rituel que l'on accomplit symboliquement en même temps ou à la place de la parole (si l'on en croit l'alternative entre geste et parole énoncée par Ulysse vis-à-vis de Nausicaa): "toucher les genoux" et/ou dire "je touche tes genoux"

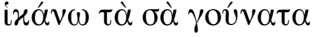

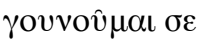

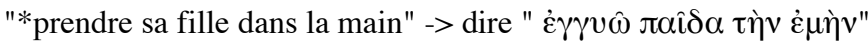

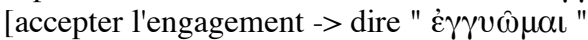

Rappelons que le baptême souvent pris pour exemple par Austin, qu'il s'agisse d'un bateau ou d'une personne, vient aussi du grec, et d'un verbe qui signifie à l'origine "plonger (dans l'eau)" au sens transitif. Il s'agit encore du geste originel que Saint Jean dit justement le Baptiste accomplit pour la première fois avec le Christ.

Il faut dès lors moduler les formulations tranchantes d'Austin sur le rôle non descriptif du performatif, pour parler au moins du point de vue diachronique d'une description originelle d'un geste, qui disparaît ou a tendance à disparaître quand le geste s'incorpore à la parole au point qu'elle n'ait plus besoin du tout de son support (le baptême d'un enfant ne suppose plus qu'on le plonge dans l'eau du baptistère, mais une goutte d'eau symbolique suffit).

Nous avons évité de nous engager dans la question du locutoire et de l'illocutoire, les langues anciennes ne permettant guère de telles spéculations, qui ne font d'ailleurs pas partie du noyau le plus intéressant et le plus fécond de la théorie. Mais le fait que la supplication, la promesse, l'engagement, soient très proches des catégories les plus souvent prises comme exemples dans Quand dire c'est faire semble garantir la validité des analyses proposées. Le fait que les langues dont nous nous occupons, à défaut de locuteurs capables de les valider, fournisse l'appui d'une histoire dans la longue durée peut être considérée comme une forme de compensation.

\section{Bibliographie $^{60}$}

Anscombre J.-C. - Létoublon F. \& Pierrot A. 1987 "Speech Act Verbs, Linguistic Action, Verb and Delocutivity", in Verschueren 45-67.

Arend W. 19333 Die typischen Szenen bei Homer, Berlin, Hermes Suppl.

Aubriot-Sévin D. 1992 Prière et conceptions religieuses en Grèce ancienne jusqu'à la fin $d u V^{e}$ siècle av. J.-C., Lyon, Maison de l'Orient méditerranéen, CMO 22.

Austin J.L. 1962 How to do things with words, Oxford, Clarendon Press. Tr. fr. Quand dire c'est faire, Paris, 1970.

Benveniste E. 1954 "Problèmes sémantiques de la reconstruction", Word 10, 251-264, repr. in 1966, 289-307. 258-266.

- 1958 "De la subjectivité dans le langage", Journal de psychologie, 0000, repr. in 1966,

- 1958 "Les verbes délocutifs", Mélanges Spitzer, 57-63, repr. in 1966, 277-285.

- 1963 "La philosophie analytique et le langage", Les études philosophiques 1, 3-11, repr. in $1966,267-276$.

- 1966 Problèmes de linguistique générale, Paris, Gallimard.

Bourdieu P. 1975 "Le langage autorisé. Note sur les conditions sociales de l'efficacité du discours rituel", Actes de la recherche en sciences sociales 5-6, 183-190.

- 1982 Ce que parler veut dire. L'économie des échanges linguistiques, Paris, Fayard.

\footnotetext{
${ }^{60}$ La bibliographie ne figure pas dans l'édition imprimée. Je pense utile de la conserver pour cette version.
} 
Burkert W. 1996 Creation of the Sacred. Tracks of Biology in early Religions, Cambridge Mass., Harvard Univ. Pr.

Cassin B., Rosier-Catach I. et Laugier S. 2004 "Acte de langage", 11-21.

- (éd.) 2004 Vocabulaire européen des philosophies, Paris, Seuil.

Cléro J.-P. et Laugier S. 2004 "Anglais. III Le paradigme austinien" in Cassin (éd.) 2004, 95-99.

Corlu A. Recherches sur les mots relatifs à l'idée de prière, d'Homère aux tragiques,

Paris, Klincksieck.

Crotty K. 1994 The Poetics of Supplication. Homer's Iliad and Odyssey, Ithaca, Cornell

University Press, Myth and Poetics.

Cuisenier J. 2006 Penser le rituel, Paris, PUF.

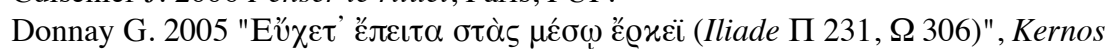

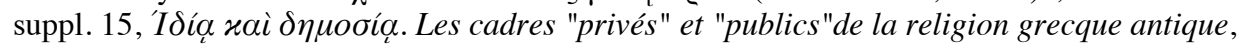
édité par Véronique Dasen \& Marcel Piérart, Liège, CIERGA, p. 1-11.

Ducrot O. 1972 Dire et ne pas dire, Paris.

-1980 Les mots du discours, Paris.

Giordano M. 1999a La Supplica. Rituale, istituzione sociale e tema epico in Omero, Napoli, AION 3.

Giordano M. 1999b La parola efficace. Maledizioni, giuramenti e benedizioni nella

Grecia arcaica, Pisa-Roma, Bibllioteca di QUCC 7.

Gould J. 1973 "Hiketeia", JHS 93, 74-103, repr. in 2001, 22-77.

- 2001 Myth, Ritual, Memory, and Exchange. Studies in Greek Literature and Culture,

Oxford, OUP.

Latraverse F. 1987 La pragmatique. Histoire et critique, Bruxelles.

Létoublon F. 1980 "Le vocabulaire de la supplication en grec: performatif et dérivation délocutive", Lingua 52, 325-336.

- 1985 Il allait, pareil à la nuit. Les verbes de mouvement en grec: supplétisme et aspect verbal, Paris, Klincksieck.

- 1986 "Comment faire des choses avec des mots grecs. Les actes de langage dans la langue grecque", in Philosophie du langage et grammaire dans l'Antiquité, Cahiers de philosophie ancienne 5, Cahiers du groupe de recherches sur la philosophie et le langage 67, Bruxelles-Grenoble, 67-90.

- 1989 "Le serment fondateur", Mètis 4, 101-115.

- 1991 "Promisi per iocum", in New Studies in Latin Linguistics, ed. Robert Coleman, Amsterdam, Benjamins (Studies in Language Companion Ser. 21), 163-185.

- 2003 "«Promettre» et les jeux de langage chez Plaute", Lalies 23, 191-203.

Lyons J. 1970 Linguistique générale. Introduction à la linguistique théorique, Paris, Larousse.

- 1977 Semantics, Cambridge, CUP.

Michaels A. "How do you do? Some preliminary thoughts on a grammar of ritual", texte préparatoire au colloque de Heidelberg Ritualdynamik, sept. 2008.

Naiden F.S. 2007 Ancient Supplication, Oxford, OUP.

Onians R.B. 1999 Les origines de a pensée européenne sur le corps, l'esprit, l'âme, le monde, le temps et le destin, Paris, Seuil, trad. par B. Cassin, A. Debru, M. Narcy (éd. orig. en anglais, Cambridge, 1951).

Pulleyn S. 1997 Prayer in Greek Religion, Oxford, Clarendon Press.

Récanati F. 1981 Les énoncés performatifs, Paris, Minuit.

Reinhardt K. 1961 Die Ilias und ihr Dichter, hsgb. U. Hölscher, Göttingen, Vandenhœek \& Ruprecht.

Rudhardt J. $1992^{2}$ Notions fondamentales de la pensée religieuse et actes constitutifs $d u$ culte dans la Grèce classique, Paris, Picard.

Searle J. 1972 Les actes de langage. Essai de philosophie du langage, Paris, Hermann, coll. Savoir (publ. orig. en anglais, Cambridge, 1969, Speech Acts). 
Sittl C. 1890 Die Gebärden der Griechen und Römer, Leipzig.

Vandendorpe C. 1999 "Rhétorique de Derrida", Littératures 19, 169-193.

Verschueren J. 1987 Linguistic Action: Some Empirical-Conceptual Studies, Series Advances in Discourse Process XXIII, Norwood. 